\title{
First direct comparison of nondisrupting neutron star-black hole and binary black hole merger simulations
}

\author{
Francois Foucart, ${ }^{1}$ Luisa Buchman, ${ }^{2}$ Matthew D. Duez, ${ }^{3}$ Michael Grudich,${ }^{1,4}$ Lawrence E. Kidder, ${ }^{5}$ \\ Ilana MacDonald, ${ }^{1,6}$ Abdul Mroue, ${ }^{1}$ Harald P. Pfeiffer, ${ }^{1}$ Mark A. Scheel, ${ }^{2}$ and Bela Szilagyi ${ }^{2}$ \\ ${ }^{1}$ Canadian Institute for Theoretical Astrophysics, University of Toronto, Toronto, Ontario M5S 3H8, Canada \\ ${ }^{2}$ Theoretical Astrophysics 350-17, California Institute of Technology, Pasadena, California 91125, USA \\ ${ }^{3}$ Department of Physics \& Astronomy, Washington State University, Pullman, Washington 99164, USA \\ ${ }^{4}$ Memorial University of Newfoundland, St. John's, Newfoundland A1C 5S7, Canada \\ ${ }^{5}$ Center for Radiophysics and Space Research, Cornell University, Ithaca, New York 14853, USA \\ ${ }^{6}$ Department of Astronomy \& Astrophysics, University of Toronto, Toronto, Ontario M5S 3H5, Canada
}

(Received 30 July 2013; published 9 September 2013)

\begin{abstract}
We present the first direct comparison of numerical simulations of neutron star-black hole and black holeblack hole mergers in full general relativity. We focus on a configuration with nonspinning objects and within the most likely range of mass ratio for neutron star-black hole systems $(q=6)$. In this region of the parameter space, the neutron star is not tidally disrupted prior to merger, and we show that the two types of mergers appear remarkably similar. The effect of the presence of a neutron star on the gravitational wave signal is not only undetectable by the next generation of gravitational wave detectors, but also too small to be measured in the numerical simulations: even the plunge, merger and ringdown signals appear in perfect agreement for both types of binaries. The characteristics of the post-merger remnants are equally similar, with the masses of the final black holes agreeing within $\delta M_{\mathrm{BH}}<5 \times 10^{-4} M_{\mathrm{BH}}$ and their dimensionless spins within $\delta \chi_{\mathrm{BH}}<10^{-3}$. The rate of periastron advance in the mixed binary agrees with previously published binary black hole results, and we use the inspiral waveforms to place constraints on the accuracy of our numerical simulations independent of algorithmic choices made for each type of binary. Overall, our results indicate that nondisrupting neutron star-black hole mergers are exceptionally well modeled by black hole-black hole mergers, and that given the absence of mass ejection, accretion disk formation, or differences in the gravitational wave signals, only electromagnetic precursors could prove the presence of a neutron star in low-spin systems of total mass $\sim 10 M_{\odot}$, at least until the advent of gravitational wave detectors with a sensitivity comparable to that of the proposed Einstein Telescope.
\end{abstract}

DOI: 10.1103/PhysRevD.88.064017

PACS numbers: 04.25.dg, 04.30.Db, 04.40.Dg

\section{INTRODUCTION}

Mergers of black holes and neutron stars are expected to be among the main sources of gravitational wave signals detectable by the next generation of gravitational wave detectors (Advanced LIGO [1], Advanced VIRGO [2], KAGRA [3]), as well as by proposed 'third generation' ground-based detectors such as the Einstein Telescope [4]. Being able to associate a detected gravitational wave signal with a given type of binary system (binary black holes $(\mathrm{BBH})$, binary neutron stars (BNS) or neutron star-black hole (NSBH) binary) is an important way to gain useful insights into the formation mechanisms of compact binaries. However, even for the binary parameters for which the types of objects involved have the largest effects, this is generally a difficult task. In this paper, we will show through the first direct comparison of numerical simulations of NSBH and BBH mergers that for configurations in which the tidal field of the black hole is not strong enough to disrupt the neutron star, these two types of mergers are remarkably similar - so much so that, even numerically, the differences in the gravitational waveform, orbital evolution, and characteristics of the final remnant cannot be resolved.

The simplest method to associate a gravitational wave event with a given type of binary relies on the determination of the mass of the compact objects, as well as the assumption of a given maximum mass for neutron stars above which all observed objects are expected to be black holes. This works for objects which are either clearly too light to be black holes or too heavy to be neutron stars. In the context of the Advanced LIGO detector, Hannam et al. [5] have recently studied which ranges of the measured chirp mass (the combination of the masses of the two objects which is most accurately measured from the gravitational wave signal) can be unambiguously associated with each type of binary. One limitation of this method, however, is that even if the gravitational wave signals were strong enough for the individual masses to be determined with high accuracy, compact objects would still be determined to be either black holes or neutron stars based solely on our preconception for the mass range in which neutron stars and black holes exist. It will never allow us to determine, for example, whether a $2.5 M_{\odot}$ object is a very massive neutron star or a very light black hole. 
More direct evidence of the presence of a neutron star is the detection of an electromagnetic counterpart to the gravitational wave signal, either before the disruption of the neutron star (see e.g. [6-8]), after the merger (e.g. gamma-ray burst, kilonova, see $[9,10]$ for a review of these electromagnetic signals and of their detectability), or, for BNS, during the ejection of relativistic material from the contact layer between the two stars [11]. The exact conditions under which premerger signals can be emitted are not, at this point, very well understood. The other signals could be fairly common in binary neutron star mergers, although potentially difficult to detect for most events (gamma-ray bursts are expected to be strongly beamed, while isotropic counterparts are likely to be too faint to be detected for the farthest binaries observable by Advanced LIGO). For NSBH binaries, recent numerical simulations have shown that for the most likely black hole masses $\left(M_{\mathrm{BH}} \sim 7 M_{\odot}-10 M_{\odot}[12,13]\right)$, the neutron star will only be disrupted by the tidal field of the black hole for rapidly rotating black holes $[14,15]$. For lower mass black holes, tidal disruption is significantly easier (see $[16,17]$ for reviews of numerical simulations of NSBH mergers). Given that post-merger electromagnetic signals cannot be emitted unless the neutron star is disrupted during the merger, this poses strong constraints on the binary parameters for which we can hope to prove the presence of a neutron star from post-merger electromagnetic observations.

Finally, measuring the effect of tides and, for NSBH binaries, of the disruption of the neutron star on the gravitational wave signal can allow us to distinguish black holes from neutron stars in merging binaries. For BNS, recent studies $[18,19]$ suggest that these effects will be measurable for a significant fraction of the Advanced LIGO events. For NSBH binaries, this would only be possible for very low mass black holes [20], or very close events with rapidly rotating black holes (at most $\sim 1 \%$ of the Advanced LIGO events with black hole spin $\chi_{\mathrm{BH}} \sim 0.9$ and mass $M_{\mathrm{BH}} \sim 10 M_{\odot}$ ) [21].

For black holes in the most likely range of masses, tidal effects during inspiral are expected to be orders of magnitude smaller [22,23]. The effects of the presence of a neutron star on the plunge, merger, and characteristics of the post-merger remnant have not, however, been studied in much detail. In terms of waveform analysis, the most complete study was performed by Lackey et al. [20], where a large number of neutron star-black hole waveforms (all for black hole masses $M_{\mathrm{BH}} \leq 7 M \odot$ ) was presented and compared with analytical approximations to black hole-black hole waveforms. Their results provide us with remarkable predictions for the effects of the equation of state of the neutron star on the gravitational wave signal emitted by low mass neutron star-black hole mergers, and their detectability by Advanced LIGO and the Einstein Telescope. As for the expected characteristics of the final black hole, a fit to the results of numerical simulations was recently proposed by Pannarale [24]. In both cases, the results resolve the effects of the presence of a neutron star in the regimes in which these effects are the largest (low mass or rapidly spinning black holes). The various analytical approximations used to cover the parameter space efficiently (numerical fits, use of approximate analytical waveforms) however dominate the error in the regime of low-spin black holes in the most realistic mass range (and, in the case of [20], the Fisher matrix analysis applied to the waveforms breaks down when estimating errors in the determination of the neutron star radius for high mass ratio systems, as discussed in Sec. IV D).

In this paper, we provide the first direct comparison between numerical simulations of NSBH and BBH mergers, and investigate in more detail the effect of the presence of a neutron star during a merger with a nonspinning black hole of mass $M_{\mathrm{BH}}=6 M_{\mathrm{NS}} \sim 8 M_{\odot}$. We show that, in every observable quantity that we considered, the two simulations are in remarkable agreement, to very high accuracy. In particular, the periastron advance, merger waveform and post-merger characteristics of the system are shown to be impossible to distinguish within the errors of the numerical simulations (and, a fortiori, identical as far as gravitational wave detectors are concerned). Even for third generation gravitational wave detectors, the presence of a neutron star would influence the gravitational waveform mainly through a tiny phase shift accumulated during the inspiral, while the merger and ringdown would provide almost no additional information.

We also use these simulations to obtain a verification of the accuracy of our code during inspiral, independent of the different algorithmic choices (gauge, grid setup,...) made in NSBH and BBH simulations. The inspiral waveforms at our highest numerical resolution agree within a phase accuracy $\delta \phi \lesssim 0.1 \mathrm{rad}$, lower than the expected numerical errors (and significantly larger than the dephasing due to tides within the neutron star). Our results also show that the rate of periastron advance in NSBH simulations is compatible with previously published results for BBH systems [25], and we verify that tidal effects in the neutron star are locally resolved and evolve as expected with the binary separation.

\section{NUMERICAL SETUP}

We simulate BBH and NSBH binaries using the SPEC code, developed by the SXS collaboration [26]. We use a first-order [27] generalized harmonic formulation of Einstein's equations [27-30] that are evolved with a multidomain pseudospectral method. The evolution region is divided into "subdomains" whose geometry (and spectral basis) is adapted to the expected symmetries of the system in each region (spheres close to the central objects and at large distance from the binary, distorted cubes and cylinders elsewhere). For NSBH mergers, the general relativistic equations of hydrodynamics are evolved in conservative 
form on a separate finite difference grid, using high resolution shock-capturing methods [31]. A more detailed description of the numerical methods used for recent evolutions of NSBH binaries with the SPEC code and of the grid structure used for the evolution of Einstein's equations can be found in Foucart et al. [21].

All simulations presented in this paper consider binaries of mass ratio $q=6$. Both objects are initially nonspinning. The BBH results were first published as part of a series of nonspinning BBH simulations by Buchman et al. [32], where more details can be found on the numerical setup used. The case considered here covers 43 gravitational wave cycles (measured up to the peak of the dominant mode of the gravitational waveform, $h_{22}$ ) at very low eccentricity (initially, $e=4 \times 10^{-5}$ ). The NSBH results are presented here for the first time. Although they represent the longest simulation of a NSBH merger published so far, they are still significantly shorter than their BBH counterparts ( 25 cycles), and have a higher eccentricity $(e<0.005$, see Sec. III for a discussion of the eccentricity). The neutron star fluid is modeled by a $\Gamma$-law equation of state

$$
\begin{gathered}
P=\kappa \rho_{0}^{\Gamma}+\rho_{0} T \\
\epsilon=\frac{1}{\Gamma-1} \frac{P}{\rho_{0}},
\end{gathered}
$$

where $P$ is the pressure, $\epsilon$ the internal energy, $\rho_{0}$ the baryon density and $T$ a quantity related to the temperature of the gas (i.e. $\rho_{0} T$ is the thermal pressure). We choose $\Gamma=2$ and $\kappa=92.12$, which for a star of gravitational mass $M_{\mathrm{NS}}=1.4 M_{\odot}$ leads to a compactness $C_{\mathrm{NS}}=$ $M_{\mathrm{NS}} / R_{\mathrm{NS}}=0.156$ (i.e. $R_{\mathrm{NS}}=13.3 \mathrm{~km}$ ). ${ }^{1}$ This radius is at the upper end of the current estimates for neutron star radii $[33,34]$. We generate constraint satisfying initial data using a spectral elliptic solver [35], solving for a quasiequilibrium state through the iterative procedure described in Foucart et al. [36]. Low-eccentricity orbits are obtained by evolving the binary for $\sim 3$ orbits, and using the measured eccentricity of the simulation to obtain an improved guess for the initial orbital and radial velocity of the binary, as described in Pfeiffer et al. [37]. One of these intermediate simulations (with $e \sim 0.024$ ) was evolved for $\sim 8$ orbits to allow us to measure the advance of the periastron (as this measurement cannot be made accurately on a low-eccentricity binary).

We run the NSBH inspirals at 3 different fixed resolutions (spectral resolution $N_{\mathrm{sp}}^{1 / 3}=57,64,72$, and finite difference resolution $\left.N_{\mathrm{fd}}^{1 / 3}=100,120,140\right)$. Additionally, we run a 4th simulation using an adaptive choice of the spectral resolution, where the number of basis functions over which we expand the solution in each of the subdomains forming

\footnotetext{
${ }^{1}$ Throughout this article, we use the convention $G=c=1$, unless units are explicitly mentioned.
}

our numerical grid is chosen adaptively, so that the truncation error of the spectral expansions of the metric components and of their spatial derivatives are below $2 \times 10^{-6}$ close to the black hole and neutron star, and $2 \times 10^{-4}$ far away from the compact objects. This offers significantly higher accuracy throughout the inspiral, at a lower cost $\left(N_{\mathrm{sp}}^{1 / 3} \sim 63\right.$ during most of the evolution, with a peak at $N_{\mathrm{sp}}^{1 / 3}=73$ during the relaxation of the initial data). This additional simulation was performed to test that some unphysical effects visible at early times in the other simulations (see Sec. III) converged away when the spectral resolution was high enough (this simulation also used $N_{\mathrm{fd}}^{1 / 3}=100$ on the finite difference grid, as our tests show that, for this configuration, the finite difference resolution does not significantly influence the inspiral results). The adaptive choice of the spectral resolution is also used for all 4 simulations during the plunge/merger phases, with truncation error of $10^{-4}, 7 \times 10^{-5}, 5 \times 10^{-5}$ for the runs using fixed resolution during the inspiral, and $10^{-4}$ for the simulation which used the adaptive method from the beginning of the simulation. ${ }^{2}$

It is also worth noting that different gauges have been used for each type of simulation. In the generalized harmonic formulation of Einstein's equations, the coordinates $x^{b}$ evolve according to

$$
g_{a b} \nabla^{c} \nabla_{c} x^{b}=H_{a}\left(x, g_{a b}\right),
$$

where $g_{a b}$ is the spacetime metric, and the $H_{a}$ are freely specifiable functions of both the coordinates and the metric. In the three "fixed resolution" runs, we fixed $H_{a}$ to its initial value in the coordinate frame comoving with the binary. For the last simulation ('adaptive' run) and the $\mathrm{BBH}$ simulations, we used the harmonic gauge $H_{a}=0$, with a smooth transition of $H_{a}$ from its initial value over a short damping timescale $t_{\mathrm{damp}}=50 \mathrm{M}$ at the beginning of the simulation (where $M$ is the total mass of the system). Numerical tests on the early part of the evolution of NSBH binaries have shown that the harmonic gauge performs slightly better than the frozen gauge, although that change makes a significantly smaller difference than the use of the adaptive grid resolution. The harmonic gauge is also theoretically more satisfactory, as it makes the evolution of the coordinates at late times independent of the initial configuration. Its only drawback is that, at times $t \sim t_{\mathrm{damp}}$, the coordinate radius of the apparent horizon of the black hole decreases rapidly. The SPEC code requires the excision of a region inside of the black hole, and maintains the boundary of that region within the apparent horizon of the black hole. This is naturally more difficult if the apparent horizon is

\footnotetext{
${ }^{2}$ The merger in this case was only performed to extract the gravitational waveform at large radii. Given the spectral and finite difference resolutions used during merger, the post-merger results obtained from this simulation are not expected to be more accurate than those of the low resolution simulation.
} 
TABLE I. Post-merger properties of the black hole. $M_{\mathrm{BH}}^{f}$ is the final mass of the black hole, $M=M_{\mathrm{BH}}+M_{\mathrm{NS}}$ is the total mass of the system at infinite separation, $\chi_{\mathrm{BH}}=J_{\mathrm{BH}} / M_{\mathrm{BH}}^{2}$ is the dimensionless spin and $v_{\text {kick }}$ the kick velocity, as computed from the gravitational wave emission. We also give the initial $\mathrm{ADM}$ energy of the system $E_{\mathrm{ADM}}^{i}$, and the energy emitted in gravitational waves $E_{\mathrm{GW}}$ over the course of the simulation. NsBh:L0, NsBh:L1 and NsBh:L2 are the 3 simulations using fixed resolution (low, medium and high) during the inspiral, while NsBh:AMR used the adaptive choice of the spectral resolution.

\begin{tabular}{lccccc}
\hline \hline & $M_{\mathrm{BH}}^{f} / M$ & $\chi_{\mathrm{BH}}^{f}$ & $v_{\text {kick }}(\mathrm{km} / \mathrm{s})$ & $E_{\mathrm{ADM}}^{i} / M$ & $E_{\mathrm{GW}} / M$ \\
Bbh & 0.9855 & 0.3725 & $118( \pm 6)$ & 0.9960 & 0.0104 \\
\hline NsBh:L0 & 0.9832 & 0.3737 & 107 & 0.9953 & 0.0098 \\
NsBh:L1 & 0.9856 & 0.3727 & 105 & 0.9953 & 0.0098 \\
NsBh:L2 & 0.9854 & 0.3731 & 109 & 0.9953 & 0.0098 \\
NsBh:AMR & 0.9854 & 0.3726 & 109 & 0.9953 & 0.0099 \\
\hline \hline
\end{tabular}

moving rapidly on the grid, and made the use of the harmonic gauge impractical in NSBH simulations until recent improvements to the control system keeping the apparent horizon in place [38]. During mergers, $H_{a}$ is chosen according to the "damped harmonic" prescription described by Szilagyi et al. [39].

Due to these differences in the choice of the gauge functions $H_{a}$, the coordinate evolution of the binary can appear quite different for the various simulations. However, we will show that all simulations agree remarkably well on more gauge-independent quantities.

\section{ACCURACY}

For the purpose of this comparison between $\mathrm{BBH}$ and NSBH results, the BBH simulations can generally be considered as an accurate representation of the exact solution for BBH mergers. Whether in terms of the phase and amplitude errors in the gravitational waveform, the orbital eccentricity, or the properties of the final black hole, BBH simulations are indeed at least an order of magnitude more accurate than their NSBH counterparts. A detailed discussion of the numerical errors in the $\mathrm{BBH}$ results can be found in Buchman et al. [32]. The error in the BBH and NSBH simulations are only of comparable magnitude when the uncertainty due to the extrapolation of the waveform to spatial infinity and the representation of the waveform as a finite sum of spherical harmonic modes induce errors of the same order as the numerical error due to the use of finite resolution. Among the quantities discussed in this section, this is only the case for the recoil velocity of the final black hole and the total energy emitted in gravitational waves.

Although the NSBH simulations have significantly larger errors than the BBH simulations, they are nonetheless very accurate by the standard of general relativistic simulations of compact mergers with matter. The mass and spin of the final black hole, listed in Table I, converge to an accuracy of $\sim 5 \times 10^{-4} M$ for the mass and $\sim 10^{-3}$ for the dimensionless spin (for the $\mathrm{BBH}$ case, these errors are $5 \times 10^{-5} \mathrm{M}$ and $10^{-4}$, respectively). The error in the determination of the recoil velocity imparted to the black hole is mostly due to the extrapolation of the waveform to infinity, and is $\sim 10 \mathrm{~km} / \mathrm{s}$, while the energy emitted in gravitational waves is accurate to $10^{-4} M$ (for both the $\mathrm{BBH}$ and the NSBH simulations). Given that, at the end of the simulation, there is no matter left outside of the black hole, we can also check conservation of energy during the simulation, which requires

$$
E_{\mathrm{ADM}}^{i}=E_{\mathrm{GW}}+M_{\mathrm{BH}}^{f},
$$

where $E_{\mathrm{ADM}}^{i}$ is the ADM mass of the binary at the initial time, $E_{\mathrm{GW}}$ is the energy emitted in gravitational waves, and $M_{\mathrm{BH}}^{f}$ is the final mass of the black hole. This equality is satisfied within the error in the final value of the black hole mass.

A commonly used measure of the accuracy of a numerical simulation in the generalized harmonic gauge is the normalized constraint violation $\|C\|$, which measures the relative amplitude of the modes violating the generalized harmonic gauge constraint, as well as additional constraints introduced in the reduction of Einstein's equations to a set of first-order differential equations. An exact definition of $\|C\|$ can be found in Eq. (71) of [40]. In Fig. 1, we show the evolution of $\|C\|$ before the plunge of the neutron star into the black hole. From this figure, we can clearly see the advantage of choosing adaptively the number of basis functions used in the spectral decomposition of each of the subdomains forming our numerical grid: constraint violations are then $\sim 5$ times smaller than for the high-resolution run, while the number of grid points

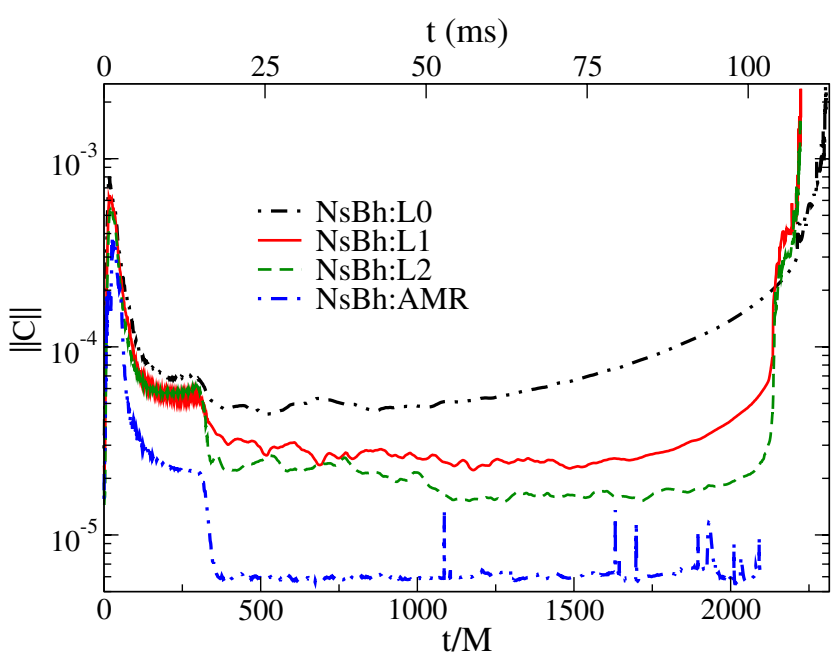

FIG. 1 (color online). Normalized constraint violation during the 4 NsBh inspirals. $\|C\|$ is defined as in Eq. (71) of [40]. The top axis (in $m s$ ) assumes $M_{\mathrm{NS}}=1.4 M_{\odot}$. 


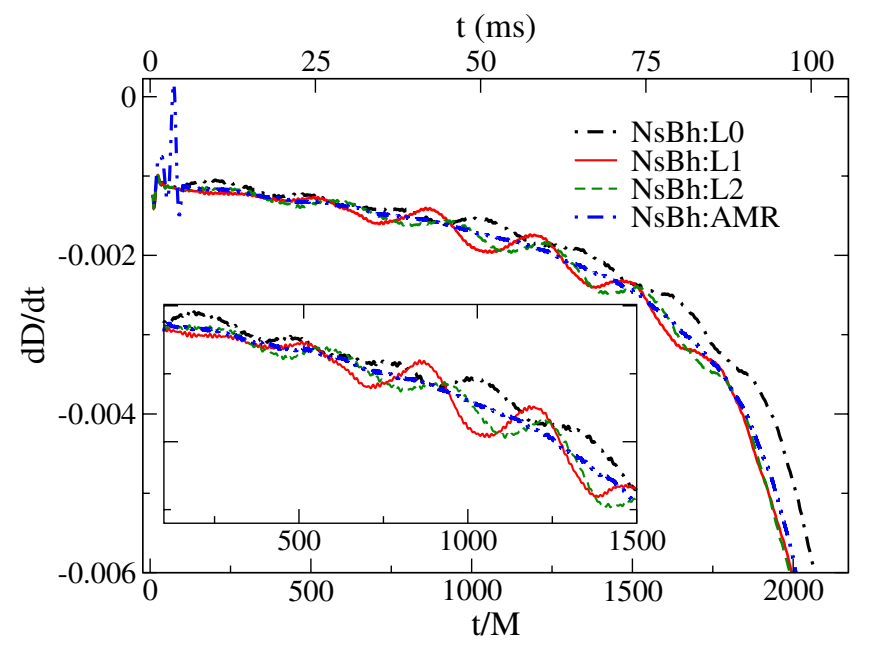

FIG. 2 (color online). Evolution of the time derivative of the coordinate separation between the center of the black hole and the center of the neutron star for the $4 \mathrm{NsBh}$ simulations. The inset zooms on the early time behavior.

is nearly the same as for the medium-resolution run. Additionally, at early times $(t<300 M)$, the fixed resolution runs are not in the convergent regime, and convergence remains slow until $t \sim 1000 M$.

Most global quantities are largely unaffected by these issues. In particular, errors in the final characteristics of the system presented in Table I are mostly determined by the resolution during the merger, where numerical errors are significantly larger (at the highest resolution, $\|C\| \sim$ 0.001-0.01 during merger). However, a subtle effect of the numerical error at early times in the fixed resolution simulations is the evolution of the eccentricity. Figure 2 shows the evolution of the time derivative of the coordinate distance $D$ between the centers of the compact objects. The 3 fixed resolution simulations appear to converge towards the simulation using adaptive grid choices, but convergence is fairly slow, and the numerical error largely appears as a growth of the eccentricity, from $e<0.001$ at early times to $e \sim 0.005$ for $t \sim 1000 M$. In the more accurate simulation using adaptive grid choices, this effect is entirely removed. At the level of accuracy obtained in these simulations, this effect is enough to affect the phase of the gravitational waveform and, in particular, the time at which the neutron star plunges into the black hole. This is visible in the fact that, despite visible differences in their evolution during the inspiral, the 2 highest fixed resolution simulations appear to agree extraordinarily well on the merger time-an agreement which is clearly accidental once the entire evolution is considered. Such accidental agreement is a strong warning as to the dangers of estimating numerical errors by solely comparing numerical simulations at two different resolutions-a procedure which, in this case, would lead us to significantly underestimate the error on the plunge time (and, consequently, on the gravitational wave phase at merger).

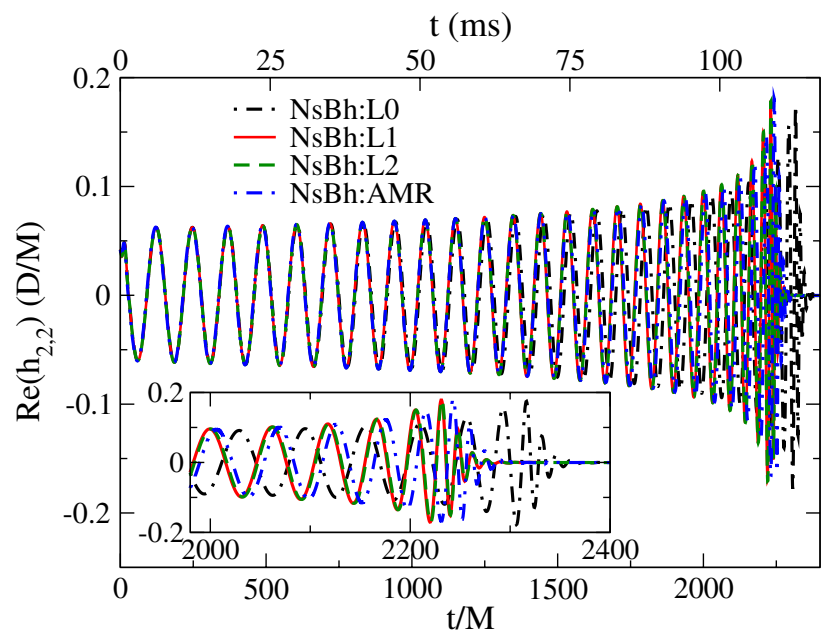

FIG. 3 (color online). Dominant $(2,2)$ mode of the gravitational waveform for the NSBH system at low, medium and high resolutions, as well as for the 'adaptive' run. The insert zooms on the time of merger.

The error on the dominant $(2,2)$ mode of the strain $h_{2,2}(t)$ can be estimated from Fig. 3, which shows the real part of $h_{2,2}$, and Fig. 4, which shows the difference in the phase of $h_{2,2}$ between the fixed resolution simulations and the "adaptive" simulation both with and without allowing the results to be shifted by an arbitrary time and phase shift. In all cases, the waveforms are obtained using 2nd order polynomial extrapolation from the waveforms measured at 20 finite radii in the interval $[100 M-275 M]$,

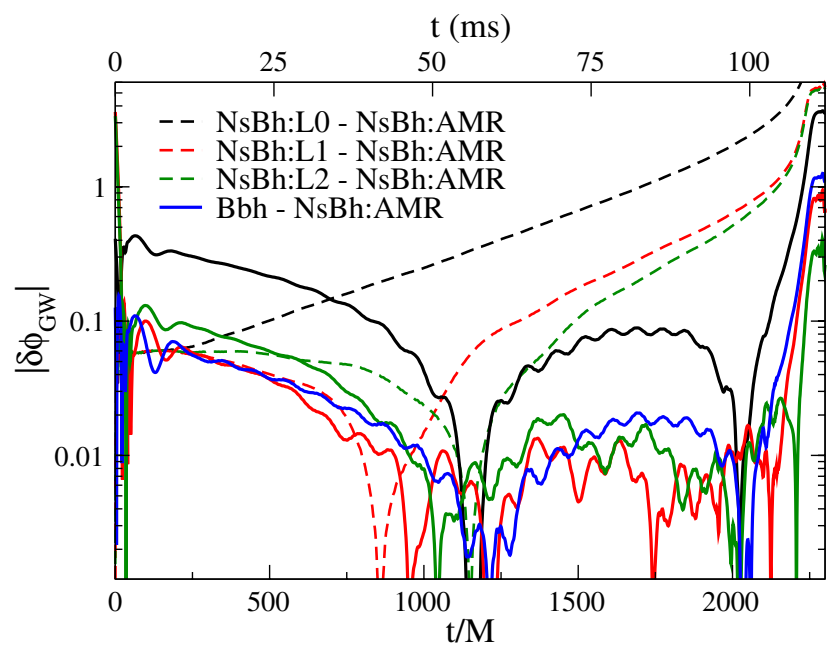

FIG. 4 (color online). Phase error in the $(2,2)$ mode of the gravitational waveform. The dashed curves show the difference between the fixed resolution simulations and the adaptive simulation without time and phase shifts, while the solid curves show the same phase differences, but after matching the waveforms in the interval $675<t / M<2175$. The solid blue curve shows the difference between the adaptive simulation and the $\mathrm{BBH}$ results (with arbitrary shifts). 
to spatial infinity, using techniques developed in [41]. For the 2 highest fixed resolutions, we see the effect of the coincident agreement in the plunge time in an apparently small error in the gravitational wave phase. A better estimate of the error is given by the difference with the adaptive simulation, which is $\sim 0.1 \mathrm{rad}$ during inspiral and $\sim 1 \mathrm{rad}$ at merger (for comparison, the error in the $\mathrm{BBH}$ simulation is $\lesssim 0.01 \mathrm{rad}$ during inspiral and $\lesssim 0.3 \mathrm{rad}$ at merger). In fact, given that the expected tidal dephasing due to finite size effects in this system is well below the numerical error (post-Newtonian estimates indicate that it should be $\lesssim 0.1 \mathrm{rad}$ without any time/phase shift for $t<2000 M[18,22,42]$, and more than an order of magnitude smaller with the shifts), the phase difference between the NSBH simulations and the BBH simulation during the inspiral is an equally valid estimate of the numerical error. And indeed, the difference between the phase of the gravitational waveform of the $\mathrm{BBH}$ simulation and that of the adaptive NSBH simulation is of the same order, and of the opposite sign, as the difference between the most accurate fixed resolution NSBH simulations and the adaptive one. Considering that at high resolution our code is only expected to be $\sim 2$ nd order convergent, obtaining the order of magnitude increase in accuracy which would be required to resolve tidal effects during the inspiral of such a NSBH binary is not a realistic objective at this point, ${ }^{3}$ nor particularly interesting consi dering that these effects cannot be resolved by the next generation of gravitational wave detectors (see Sec. IV D). Studying these effects in lower mass ratio binaries (or BNS systems) is a more realistic objective from both a numerical and observational point of view.

Finally, the error in the amplitude $A$ of the waveform is $\delta A / A \sim 2 \%$, for both the time domain waveform $h_{2,2}(t)$ and its Fourier transform $\tilde{h}_{2,2}(f)$ up to high frequency $(f \lesssim 2 \mathrm{kHz}$ ), which will allow us to compare the BBH and NSBH spectra with high accuracy. Higher-order modes have slightly larger relative errors $(\sim 5 \%$ for the first 2 subdominant modes, $h_{3,3}$ and $\left.h_{2,1}\right)$.

\section{RESULTS}

\section{A. Periastron Advance}

The trajectories of compact objects during an inspiral are intrinsically gauge-dependent, and thus difficult to compare among simulations that do not use the exact same gauge prescription. There is however one important general relativistic effect acting on the trajectories which, for nearly circular orbits and a large class of coordinate systems, is independent of these gauge choices: the rate of periastron advance of the orbit, i.e. the change in the

\footnotetext{
${ }^{3}$ Obtaining higher-order convergence in GR-Hydro codes is however possible, and has in fact been recently tested in the study of BNS systems by Radice et al. [43].
}

angular location of the periastron between two periastron passages. Explaining the periastron advance of the orbit of Mercury, first observed by Le Verrier in 1859, was one of the early successes of the theory of general relativity [44]. While this effect is small for Mercury ( $\sim 43^{\prime \prime}$ per century), it becomes very significant for compact objects close to mergers. In the context of $\mathrm{BBH}$ systems, periastron advance was measured for a number of nonspinning BBH systems by Le Tiec et al. [25], and shown to match remarkably well the predictions of the effective one-body (EOB) formalism and of the self-force theory (at least if the expansion in the mass ratio used in the self-force formalism is done using the symmetric mass ratio $\nu=$ $m_{1} m_{2} /\left(m_{1}+m_{2}\right)^{2}$ instead of $\left.q=m_{1} / m_{2}\right)$. In this section, we follow the same procedure to provide a measurement of the periastron advance for a NSBH binary of mass ratio $q=6$ and orbital eccentricity $e=0.024$, evolved for 8 orbits from an initial orbital frequency $\Omega M=0.027$.

We define the frequency of radial oscillations $\Omega_{r}=2 \pi / P$ and the average orbital frequency

$$
\Omega_{\phi}=\frac{1}{P} \int_{0}^{P} \frac{d \phi}{d t}=K \Omega_{r}
$$

where $P$ is the period of radial oscillations of the orbit and $\phi$ the orbital phase of the binary. After each radial period, the periastron will advance by an angle $\Delta \Phi=(K-1) 2 \pi$. To measure $K\left(t_{0}\right)$, we choose a time interval covering 1.5 orbital periods of the binary centered on the time $t_{0}{ }^{4}$, and fit the orbital frequency $\Omega(t)$ (measured from the coordinate trajectories) to the model

$\Omega(t)=p_{0}\left(p_{1}-t\right)^{p_{2}}+p_{3} \cos \left(p_{4}+p_{5}\left(t-t_{0}\right)+p_{6}\left(t-t_{0}\right)^{2}\right)$.

We then extract the average orbital period $\Omega_{\phi}=p_{0}\left(p_{1}-\right.$ $\left.t_{0}\right)^{p_{2}}$ and the period of radial oscillations $\Omega_{r}=p_{5}$ (as the periodic variation of $\Omega(t)$ is due to the eccentric motion of the binary). The constant $K$ is then $K_{\mathrm{NSBH}}=\Omega_{\phi} / \Omega_{r}$.

In Fig. 5, we show the result of this measurement, normalized by the value for a point particle around a Schwarzschild black hole $K_{\text {Schw }}=(1-6 x)^{-1 / 2}$ (where $x=\left(M \Omega_{\phi}\right)^{2 / 3}$ is the post-Newtonian expansion parameter) $[45,46]$. Figure 5 also shows the predictions of first-order self-force calculations [25,47], as well as the measured value for BBH systems. For the latter, we use the fit to the numerical data provided by Le Tiec et al. [25],

$$
K_{\mathrm{BBH}}=K_{\mathrm{Schw}}\left(a_{0}+a_{1}\left(M \Omega_{\phi}\right)+a_{2}\left(M \Omega_{\phi}\right)^{2}\right) .
$$

For a mass ratio $q=6$, the best-fit coefficients are $a_{0}=0.9890, a_{1}=1.071$, and $a_{2}=-57.0$ for a BBH system.

\footnotetext{
${ }^{4}$ We verified that the measured value of $K$ is insensitive to the choice of the fitting interval by using 2 and 2.5 orbital periods instead, and checking that the results are only modified at the level of the small scale oscillations visible in Fig. 5.
} 


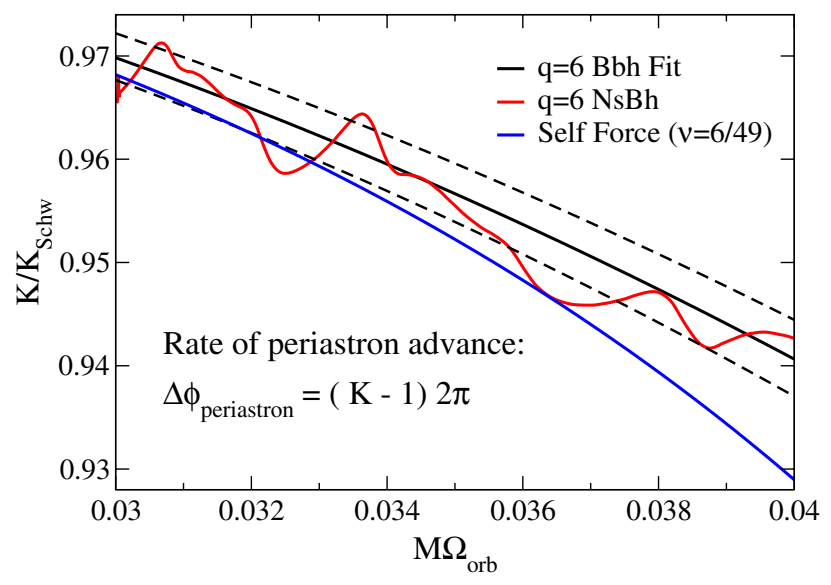

FIG. 5 (color online). Measurement of the rate of periastron advance as a function of the orbital frequency for a NSBH system of mass ratio $q=6$, compared with a fit valid for $\mathrm{BBH}$ systems [25], and the results from first-order self-force calculations $[25,47]$. All values are normalized by the rate of periastron advance for a point particle around a Schwarzschild black hole. The dashed lines show the uncertainty in the fit to the $\mathrm{BBH}$ results.

The measurement of $K_{\mathrm{NSBH}}$ provided here is clearly in good agreement with the BBH results, with

$$
\left|K_{\mathrm{NSBH}}-K_{\mathrm{BBH}}\right| / K_{\mathrm{BBH}} \leq 0.5 \% \text {. }
$$

This is comparable to the numerical error in the determination of $K_{\mathrm{NSBH}}$ (which can be estimated from the oscillations of $K_{\mathrm{NSBH}}$ ), and becomes better than the accuracy of the self-force predictions at high frequencies $\left(\Omega_{\mathrm{orb}} M \geq\right.$ 0.036). We also checked separately that a similar agreement was observed for a shorter simulation at mass ratio $q=5$ (6 orbits from an initial orbital frequency $\Omega M=0.028$, with eccentricity $e \sim 0.025$ ). The finite size of the neutron star has no measurable effect on the radial oscillations of the binary.

\section{B. Tidal distortion}

The evolution of the tidal distortion of the neutron star also follows closely the lowest-order predictions for the effect of the tidal field of the black hole. As in [21], we compute the quadrupole moments of the neutron star in the coordinate frame corotating with the binary,

$$
Q_{i j}=\int \rho\left(x_{i} x_{j}-\frac{1}{3} \delta_{i j} r^{2}\right) d V
$$

(where $x^{i}$ is a coordinate system whose origin is the center of mass of the neutron star, and oriented so that its $x$-axis points away from the black hole and its $z$-axis is parallel to the orbital angular momentum), as well as the second moment of the density

$$
I_{00}=\int \rho r^{2} d V
$$

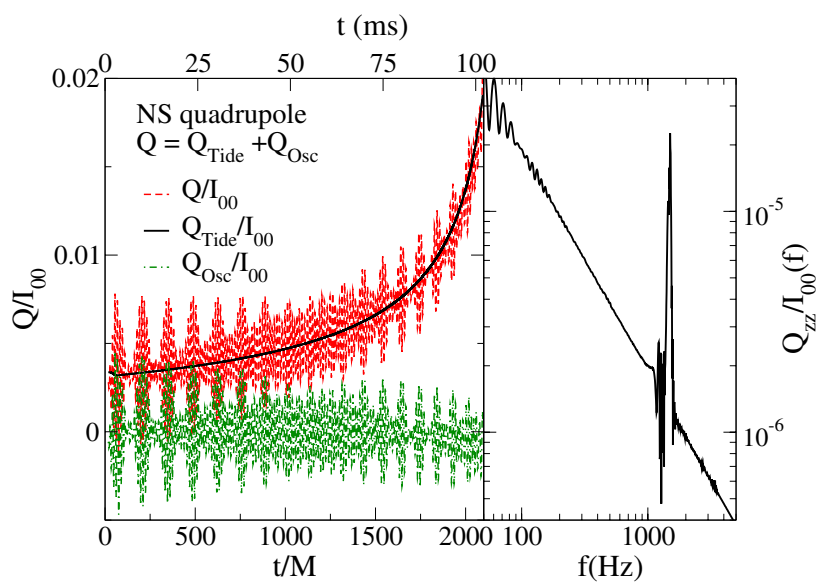

FIG. 6 (color online). Tidal quadrupole during the inspiral of the adaptive run. Left: Amplitude of the tidal quadrupole $Q$, best fit $Q_{\text {Tide }}$ to the expected evolution of the equilibrium tide (proportional to $d^{-3}$, where $d$ is the binary separation), and residual $Q_{\text {osc }}$ from that fit. Right: Fourier transform of $Q_{z z} / I_{00}$, composed of a power-law component (equilibrium tide) and an oscillatory component at $1.5 \mathrm{kHz}$ (assuming $M_{\mathrm{NS}}=1.4 M_{\odot}$ ).

where $\rho=\sqrt{g} W \rho_{0}$ and $W$ is the Lorentz factor of the fluid. We then extract the tidal part $Q$ of the quadrupolar distortion from the coordinate distortion due to the Lorentz boost given to the neutron star using the approximate method described in [21]. The results are shown in Fig. 6. The time dependence of $Q / I_{00}$ clearly has 2 main components: the expected growth of the equilibrium tide as the neutron star gets deeper into the tidal field of the black hole, which is proportional to $d^{-3}$ (where $d$ is the coordinate separation of the binary), and a slowly damped oscillatory component, due to the excitation of resonances in the neutron star in the imperfect initial data. To separate the two, we fit $Q_{\text {Tide }} / I_{00}=\alpha d^{-3}$ to find the equilibrium tidal component, and subtract $Q_{\text {Tide }}$ from the measured $Q$ to obtain the oscillatory component. The equilibrium component clearly captures the expected long term evolution of $Q$. In a previous paper [21], we had also observed a surprisingly good agreement between numerical results and the lowest-order theoretical prediction

$$
\frac{Q}{I_{00}} \sim 2 k_{2} R_{\mathrm{NS}}^{5} \frac{M_{\mathrm{BH}}}{I_{00} d^{3}},
$$

(where $k_{2}$ is the tidal Love number of the neutron star, which for the equation of state used here was computed by Hinderer [48], and $I_{00}$ is computed for an isolated neutron star). This is not the case for the simulations presented here, where the numerical value of $Q / I_{00}$ is $\sim 50 \%$ larger than the theoretical value. This is not particularly surprising: the normalization of $Q / I_{00}$ is expected to be gauge-dependent (e.g. it depends on the definition of the coordinate distance $d$ ), and the order-of-magnitude agreement found here is theoretically more reasonable than the nearly exact (and probably coincidental) agreement found in [21]. 
The high-frequency oscillations shown here were not resolved in [21], where $Q$ was only computed at a small number of discrete times. They simply appeared as a random error on the measured value of $Q$. The improved sampling rate used in this work allow us to clearly show that these deviations are not just due to errors in the method used to extract $Q$, but are instead due to the ringing of a stellar mode at $f \sim 1.5 \mathrm{kHz}$ (see the right panel of Fig. 6, in which we show the Fourier transform of $Q_{z z} / I_{00}$, the component of $Q_{i j}$ which is not affected by the transformation between the inertial and corotating coordinate systems). The amplitude of these oscillations decreases over time, albeit very slowly (by about a factor of 2 over the course of the entire simulation). This mode is excited in the imperfect quasi-equilibrium initial data, and rings without much dissipation during the inspiral. Despite the fact that the oscillatory part of $Q$ is initially of the same amplitude as the equilibrium tide, it should have a negligible effect on the evolution of the orbit and the emitted gravitational waves because, as opposed to the tidal quadrupole, it does not efficiently couple to the orbital quadrupole.

\section{Merger dynamics and final remnant}

Agreement in the rate of periastron advance between $\mathrm{BBH}$ and NSBH simulations, albeit reassuring, is not overly surprising considering that effects of the finite size of the neutron star during the inspiral (e.g. tides) are known to have only a small influence on the orbital evolution of a binary of mass ratio $q=6$. It is only during the plunge and merger that we would expect larger differences to occur, both in the properties of the final black hole and in the gravitational wave spectrum at high frequency. Instead, we find that their observable properties (gravitational waves, characteristics of the post-merger remnant,...) are surprisingly similar.

For a binary of mass ratio $q=6$ and a nonspinning black hole, we expect the neutron star to reach the innermost stable circular orbit (ISCO) of the black hole before tidal effects cause the star to overflow its Roche lobe (thus causing unstable mass transfer onto the black hole, and the disruption of the neutron star): for the neutron star of compactness $C_{\mathrm{NS}}=0.156$ considered here, a dimensionless black hole spin $\chi_{\mathrm{BH}} \gtrsim 0.6$ is expected to be required for disruption to occur before the plunge [15]. But even during the plunge, the neutron star is largely unaffected by the tidal field of the black hole: Fig. 7 shows the matter distribution as material from the neutron star begins to cross the apparent horizon of the black hole. From this gauge-dependent visualization, it appears that the distortion of the neutron star is minimal even at the point at which the largest tidal effects are expected: deviations from spherical symmetry are barely larger in the neutron star than in the black hole.

Another way to see this lack of distortion of the neutron star is to look at the accretion of material onto the black

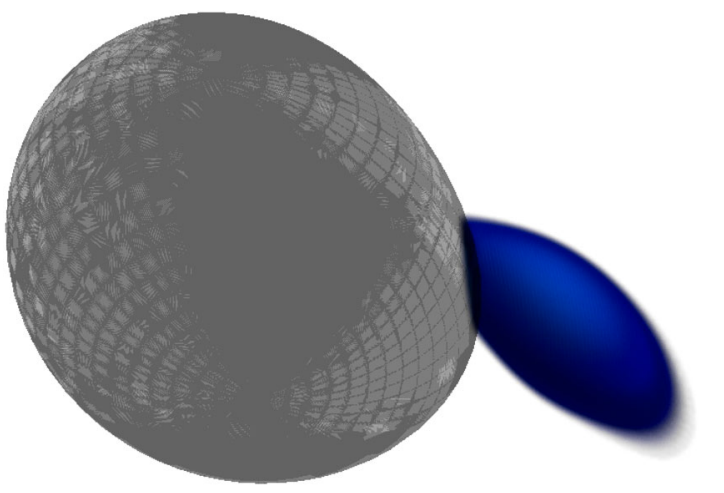

FIG. 7 (color online). Matter distribution (in blue) and location of the apparent horizon (in grey) after 5\% of the neutron star material has been accreted onto the black hole. Even at merger, the neutron star remains remarkably compact.

hole: the entire neutron star crosses the apparent horizon of the black hole within $0.5 \mathrm{~ms}$ - only a few times the lightcrossing time across the undisturbed neutron star.

But the most striking agreement between the $\mathrm{BBH}$ and the NSBH results is probably in the characteristics of the resulting black holes, summarized in Table I: the masses of the final black holes agree within an accuracy of $\delta M_{\mathrm{BH}}^{f} \lesssim$ $5 \times 10^{-4} M$, and their spins within $\delta \chi_{\mathrm{BH}}^{f} \lesssim 0.001$ ! These constraints are more than an order of magnitude tighter than existing predictions based on numerical fits to previous simulations of NSBH and BBH mergers [24]. Even the velocity kicks given to the remnants are in good agreement between the NSBH and BBH simulations, with $v_{\text {kick }} \sim 100-125 \mathrm{~km} / \mathrm{s}$ for all cases. ${ }^{5}$

For nonspinning objects, in the most likely range of black hole masses, the merger and post-merger evolution of NSBH binaries will thus look exactly identical to their BBH counterparts: no accretion disk will be formed, no matter will be ejected, and differences in the properties of the final black hole will be negligible.

This agreement occurs despite the fact that the two types of mergers have different topologies. The event horizon of a NSBH merger, which cannot be directly computed in our numerical simulations, should form a single worldtube instead of the merging event horizons ("pants" diagram) typical of BBH mergers. The marginally trapped surfaces (apparent horizons) which are followed by our code also have different geometries. In NSBH mergers, we observe a continuous evolution of the apparent horizon of the initial black hole towards the apparent horizon of the final black hole. In BBH mergers, the two apparent horizons of the initial black holes coexist on some spacelike hypersurfaces

\footnotetext{
${ }^{5}$ Note that the error in the computation of $v_{\text {kick }}$ is due in about equal amounts to the finite resolution used in the simulation and to the procedure used to extrapolate the gravitational waveform to infinity and measure from it the linear momentum emitted by the system.
} 
with an outer apparent horizon surrounding them (including on some hypersurfaces used as constant time slices in our numerical evolutions). From a geometrical standpoint, NSBH and BBH mergers are thus very different eventsbut our results show that these differences do not significantly affect the observable properties of the merger.

\section{Gravitational Waveforms}

We have just seen that, for the parameters considered here, NSBH and BBH mergers are remarkably similar in terms of orbital evolution, merger dynamics, and local properties of the post-merger remnant. The main observable counterpart to the merger of a NSBH binary, however, is the gravitational wave signal emitted during its long inspiral and eventual merger. It would thus be natural to expect that differences in their gravitational waveforms would be the easiest way to tell NSBH and BBH systems apart. In this section, we will see that although this is indeed likely to be the case, it remains an extremely challenging task which for black holes of mass $M_{\mathrm{BH}} \gtrsim$ $7 M_{\odot}$ is probably beyond the reach of the upcoming advanced gravitational wave detectors.

Recent simulations have slowly made this more apparent: if for binary neutron stars the effect of the finite size of the neutron star is expected to be detectable for a significant fraction of Advanced LIGO events [18,19] (assuming that those finite size effects can be accurately modeled, which remains an important area of research), things become significantly more complicated once NSBH mergers are considered. Finite size effects can be marginally resolved in low mass systems [20,49] or, in the most likely range of black hole masses, for at most a few percents of the events with high black hole spin $[20,21]$. This additional difficulty comes from the fact that tidal effects during the inspiral are significantly reduced for asymmetric mass ratios [22,23], while the disruption of the neutron star (which causes a sharp cutoff in the gravitational wave emission) occurs at frequencies slightly above the main Advanced LIGO band ( $f_{\text {cut }} \gtrsim 1.5 \mathrm{kHz}$ ). Unless the astrophysical population of NSBH binaries is particularly favorable for upcoming gravitational wave detectors, it is thus likely that finite size effects in NSBH binaries will only be detectable with an improved detector such as the proposed Einstein Telescope.

The details of the gravitational wave signals in the case of NSBH mergers in which the neutron star does not disrupt, and in particular their differences with signals from $\mathrm{BBH}$ mergers, remained until now largely unexplored. The best comparison to date, by Lackey et al. [20], resolves finite size effects in the low mass ratio limit, as well as the disruption of the neutron star when it occurs. However, the $\sim 30 \%$ error expected in the modeled gravitational wave spectrum at disruption, and the uncertainties in the phenomenological waveforms used as references for the $\mathrm{BBH}$ cases, do not allow for accurate direct

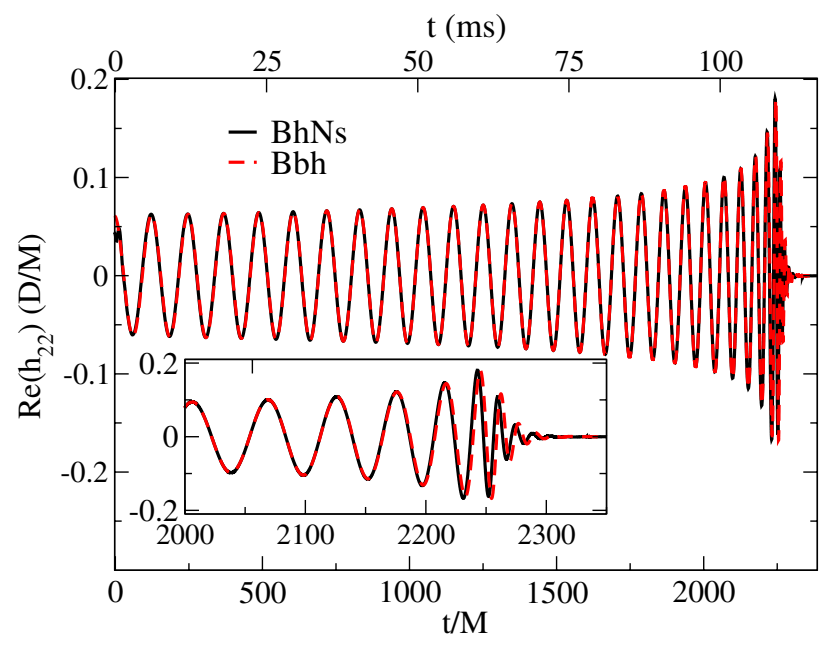

FIG. 8 (color online). Dominant $(2,2)$ mode of the gravitational waveform for the NSBH (adaptive run) and BBH mergers. The insert zooms on the time of merger. A time and phase shifts have been applied to the $\mathrm{BBH}$ waveform in order to minimize the phase difference with the NSBH results in the interval $675<t / M<2175$.

comparisons of $\mathrm{BBH}$ and NSBH results in the regime that we consider here ( $q=6$, non spinning). ${ }^{6}$ We do not, of course, expect larger differences for these nondisrupting cases. However, it was a priori unclear how much more difficult the detection of finite size effects would be for a nonspinning black hole around the peak of the black hole mass function. Indeed, even though the neutron star was expected to reach the ISCO largely undisturbed, the ISCO frequency is still relatively low $\left(f_{\mathrm{ISCO}}^{\mathrm{GW}} \sim 600 \mathrm{~Hz}\right.$ for a nonspinning black hole of mass $M_{\mathrm{BH}} \sim 8.4 M_{\odot}$ and for $q=6$, according to first order self-force calculations [51]). The plunge, merger and ringdown thus represent a larger fraction of the detectable signal, starting at a frequency well below the cutoff frequencies observed in disrupting binaries.

Alas, even after the neutron star reaches the ISCO the signal remains remarkably devoid of any imprint of the presence of a neutron star. Figure 8 shows the real part of the strain in the time domain for the BBH and NSBH mergers. The difference between the two waveforms is within the numerical error of the NSBH simulations. Figure 9 shows the spectrum of the dominant $(l=2$, $m=2$ ) mode of the gravitational wave signal as seen by an optimally oriented observer $100 \mathrm{Mpc}$ away, for both the $\mathrm{BBH}$ and NSBH systems. The two cannot be distinguished at the $\sim 2 \%$ accuracy level of our simulations, even at frequencies $f \sim 1-2 \mathrm{kHz}$ well beyond the ISCO frequency. The same is true of the two largest subdominant modes, also shown in Fig. 9.

\footnotetext{
${ }^{6}$ See also [50] for a more accurate model of the amplitude of the gravitational wave signal emitted by nonspinning NSBH binaries, but without any phase information.
} 


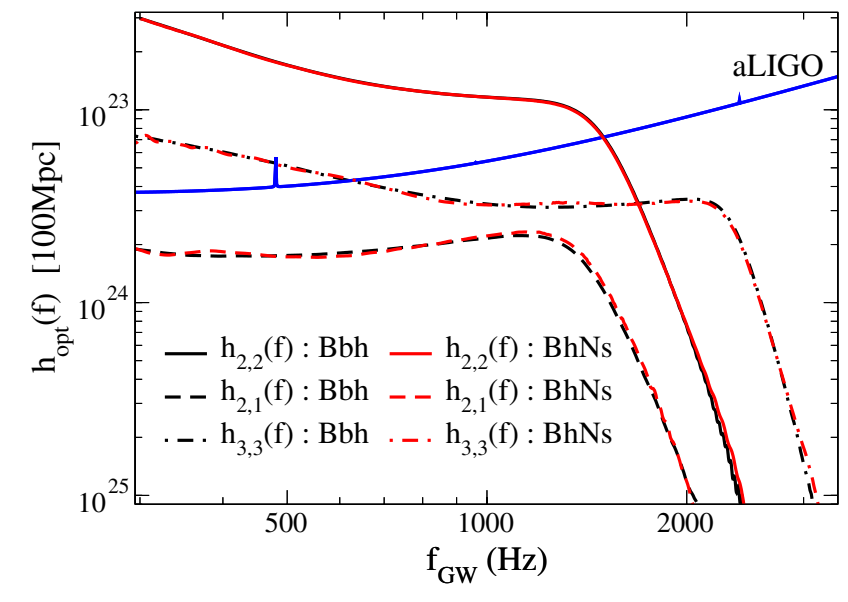

FIG. 9 (color online). Amplitude of the gravitational wave spectrum for optimally oriented binaries located at $100 \mathrm{Mpc}$. The aLIGO curve is the Zero-Detuned High Power noise curve of Ref. [52]. BBH results are shown in black and NSBH results in red for the dominant mode ( $l=2, m=2$, solid curves), and the two largest subdominant modes $(l=2, m=1$, dashed curves and $l=3, m=3$, dash-dotted curves). The amplitude $h_{\text {opt }}$ is defined as $h_{\text {opt }, l m}(f)=\sqrt{5 / 4 \pi} f^{1 / 2} h_{l m}(f)$.

From these waveforms, we can directly obtain an upper bound on the distance at which a gravitational wave detector would be able to observe the difference between a NSBH and a BBH merger, if it was only looking at the time frame covered by the numerical simulations. To do so, we define the difference $\|\delta h\|$ between two waveforms $h_{1}$ and $h_{2}$ as

$$
\|\delta h\|=\min _{\Delta t, \Delta \phi}\left(\sqrt{\left\langle h_{1}-h_{2}, h_{1}-h_{2}\right\rangle}\right)
$$

where the product $\langle g, h\rangle$ is given by

$$
\langle g, h\rangle=2 \int_{0}^{\infty} d f \frac{\tilde{g}^{*}(f) \tilde{h}(f)+\tilde{g}(f) \tilde{h}^{*}(f)}{S_{n}(f)}
$$

and we have applied to $h_{2}(t)$ a time shift $\Delta t$ and phase shift $\Delta \phi$ minimizing $\|\delta h\|$. Here $\tilde{g}(f)$ and $\tilde{h}(f)$ are the Fourier transforms of $g(t)$ and $h(t)$, and $S_{n}(f)$ is the one-sided power spectral density of the detector's strain noise, defined as

$$
S_{n}(f)=2 \int_{-\infty}^{\infty} d \tau e^{2 \pi i f \tau} C_{n}(\tau), \quad f>0,
$$

where $C_{n}(\tau)$ is the noise correlation matrix for zero-mean, stationary noise. Taking for $S_{n}(f)$ the Zero Detuned High Power spectrum from [52], which is the design sensitivity of Advanced LIGO, and limiting the integral in Eq. (13) to frequencies $f>0.3 \mathrm{kHz}$, we find the differences $\|\delta h\|$ listed in Table II. If we neglect degeneracies between the effect of the finite size of the neutron star and other parameters of the binaries, an approximate condition for the difference between two waveforms to be detectable is
TABLE II. Differences $\|\delta h\|$ between numerical waveforms computed for frequencies above $0.3 \mathrm{kHz}$. All values are computed for the Zero-Detuned High Power noise curve of the AdvLIGO detector [52], and an optimally oriented source located at $100 \mathrm{Mpc}$.

\begin{tabular}{lcccc}
\hline \hline vs & Bbh & NsBh:L0 & NsBh:L1 & NsBh:L2 \\
\hline Bbh & $\ldots$ & 0.17 & 0.08 & 0.06 \\
NsBh:L0 & 0.17 & $\cdots$ & 0.22 & 0.22 \\
NsBh:L1 & 0.08 & 0.22 & $\cdots$ & 0.04 \\
NsBh:L2 & 0.06 & 0.22 & 0.04 & $\cdots$ \\
\hline \hline
\end{tabular}

$\|\delta h\|>1$ [53]. Accordingly, our results show that, at best, differences in the merger waveforms would be observable by Advanced LIGO for optimally oriented binaries located within $\sim 10 \mathrm{Mpc}$ of the detector-or about once in a million events. Clearly, the high frequency portion of the waveform alone will be of no use to an observer attempting to determine whether a gravitational wave signal comes from a BBH or a NSBH binary.

Of course, in practice, gravitational wave detectors will not rely solely on the merger waveform. Most of the signalto-noise is accumulated at lower frequencies during the inspiral. Finite size effects during the inspiral have been estimated in the post-Newtonian formalism [18,22,42,54]. They cause NSBH and BBH systems to accumulate a small phase shift over time. For the binary parameters considered here, this phase shift is much below the phase accuracy of our code (see Sec. III), and the measured phase difference between our numerical BBH and NSBH waveforms before merger (see Fig. 4) is mostly a measure of the accuracy of our code. Nevertheless, tidal dephasing during the binary inspiral is the easiest way to measure finite size effects in the gravitational waveform for a $q=6$, nonspinning system. As in [21], we can estimate the difference $\|\delta h\|$ between the BBH and NSBH waveforms by attaching to the numerical merger a post-Newtonian inspiral, with or without tidal terms. For the post-Newtonian waveform, we use the Taylor T1 expansion, which matches numerical results very well for $\mathrm{BBH}$ at $q=6$ [55]. The postNewtonian waveform is matched to the numerical results in the frequency range $f=300-700 \mathrm{~Hz}$. Using the tidal phase shift computed in [18] for the phase difference between the NSBH and BBH post-Newtonian waveforms, we find that for our $C_{\mathrm{NS}}=0.156$ neutron star tidal effects would lead to $\|\delta h\|>1$ for optimally oriented binaries within a distance $D_{\max } \sim 100 \mathrm{Mpc}$ for the Advanced LIGO detector at design sensitivity. As the signal-to-noise ratio of such an event would be $\sqrt{\langle h, h\rangle}=63.9$, and given that a signal-to-noise ratio of 8 is required for event detection in Advanced LIGO, this represents about $0.2 \%$ of the detectable events with these binary parameters (changing the numerical resolution of the NSBH results, the frequency interval for the matching, and the choice of the post-Newtonian order used for the tidal dephasing leads to 
a relative uncertainty of $\sim 30 \%$ on $D_{\max }$, and thus of about a factor of 2 in the number of events for which the presence of a neutron star would be detectable). Taking into account degeneracies with the mass and spin of the objects generally reduces this distance by about a factor of $\sim 2-3$ [20], and thus the event rate by at least an order of magnitude.

These results might seem pessimistic when compared with the predictions obtained for nonspinning systems at an only slightly lower mass ratio $(q=5)$ by Lackey et al. [20], where the relative error on the tidal parameter $\Lambda^{1 / 5}$ is measured through a Fisher matrix analysis $(\Lambda=$ $2 / 3 k_{2} C_{\mathrm{NS}}^{-5}$ is the tidal deformability and $\Lambda^{1 / 5}$ is thus mostly proportional to the radius of the neutron star). For the neutron star considered here $\left(\Lambda^{1 / 5} \sim 3.5\right)$, Lackey et al. [20] estimate the relative error in the measurement of $\Lambda^{1 / 5}$ to be $\left(\sigma_{\Lambda^{1 / 5}} / \Lambda^{1 / 5}\right) \sim 0.45$ for an optimally oriented binary of mass ratio $q=5$ at $100 \mathrm{Mpc}$, after marginalizing over the masses of the objects and the spin of the black hole. This appears to be about 5 times more optimistic than the results presented here. The 2 results can, however, easily be reconciled if we remember that the Fisher matrix approximation is based on a first-order expansion of the waveform in the parameters $\boldsymbol{\theta}=\left\{\theta_{i}\right\}$ of the binary, i.e.

$$
\tilde{h}(\boldsymbol{\theta})=\tilde{h}\left(\boldsymbol{\theta}^{0}\right)+\frac{\partial \tilde{h}}{\partial \theta_{i}}\left(\theta_{i}-\theta_{i}^{0}\right) .
$$

The Fisher matrix is then

$$
\Gamma_{i j}\left(\boldsymbol{\theta}^{0}\right)=\left\langle\frac{\partial \tilde{h}}{\partial \theta_{i}}\left(\boldsymbol{\theta}^{0}\right), \frac{\partial \tilde{h}}{\partial \theta_{j}}\left(\boldsymbol{\theta}^{0}\right)\right\rangle
$$

and the error in the measurement of $\theta_{i}$ is $\sigma_{\theta_{i}}=\sqrt{\left(\Gamma^{-1}\right)_{i i}}$ (see [20] for more details). For relative errors of order unity, the choice of expansion parameter can critically affect the results of the Fisher matrix analysis. In particular, it is easy to see that ${ }^{7}$

$$
\frac{\sigma_{\Lambda^{1 / 5}}}{\Lambda^{1 / 5}}=\frac{1}{5} \frac{\sigma_{\Lambda}}{\Lambda},
$$

a result which is obviously exact for small deviations $\delta \Lambda$ from the true value $\Lambda_{0}$, as

$$
\left(\Lambda_{0}+\delta \Lambda\right)^{1 / 5}=\Lambda_{0}^{1 / 5}\left(1+\delta \Lambda /\left(5 \Lambda_{0}\right)+O\left(\left(\delta \Lambda / \Lambda_{0}\right)^{2}\right)\right.
$$

but not longer holds when $\left|\sigma_{\Lambda^{1 / 5}} / \Lambda^{1 / 5}\right| \lesssim 0.15$. For the configuration considered in this paper, if the Fisher matrix analysis indicates a relative error of about $45 \%$ in a measurement of $\Lambda^{1 / 5}$ for an optimally oriented binary at

\footnotetext{
${ }^{7}$ In Eq. (17), it is implicitly assumed that $\sigma_{\Lambda^{1 / 5}}$ is the result of a Fisher matrix analysis in which $\theta_{i}=\left(\Lambda^{1 / 5}, \alpha_{k}\right)$ and $\sigma_{\Lambda}$ the result of a Fisher matrix analysis for $\theta_{i}=\left(\Lambda, \alpha_{k}\right)$, where the $\alpha_{k}$ are the same additional parameters in both cases (e.g. masses, black hole spin, coalescence time and phase,...).
}

$100 \mathrm{Mpc}$, the same analysis would predict a relative error of $220 \%$ in a measurement of $\Lambda$-a sign that the local approximation used by the Fisher matrix is no longer valid. Our results indicate that the correct answer is fairly close to what one would get using a local expansion in $\Lambda$ - which is not too surprising if we remember that the tidal dephasing $\delta \Psi(f)$ scales linearly with $\Lambda$, and thus $\partial \tilde{h} / \partial \Lambda$ is nearly constant as $\Lambda$ varies (while $\partial \tilde{h} / \partial \Lambda^{1 / 5}$ vanishes as $\Lambda \rightarrow 0$ ). In fact, a Fisher-matrix analysis using $\Lambda$ as an expansion parameter will be included in an updated version of [20], with results in good agreement with the estimates presented here [56]. The Fisher matrix analysis expanding in $\Lambda^{1 / 5}$ does, however, remain perfectly valid for larger signal-to-noise ratios (i.e. either more advanced detectors, such as the Einstein Telescope, closer binaries, or low mass systems).

\section{CONCLUSIONS}

In this work, we compared numerical simulations of binary black hole and neutron star-black hole binaries with the SpEC code, focusing on a nonspinning binary within the range of mass ratio currently favored by black hole mass measurements $\left(M_{\mathrm{BH}}=6 M_{\mathrm{NS}}\right)$. Our results place strong upper limits on the difference between the 2 types of binaries in terms of the orbital and merger dynamics, the characteristics of the remnant black hole, and the gravitational wave signal emitted during the merger for NSBH mergers in which the neutron star reaches the ISCO without being tidally disrupted. Because of the expected similarity of the inspiral waveform, these simulations also provide the strongest test so far of the accuracy of the SPEC code for general relativistic hydrodynamics simulations.

In particular, we measured the rate of periastron advance during the inspiral of the NSBH binary, and found it to be in good agreement with the results of Le Tiec et al. [25] for BBH systems. We also showed that tidal effects in the neutron star are resolved locally in our simulations, and evolve as expected as the binary separation shrinks. But, as predicted by post-Newtonian estimates, they are not significant enough to cause measurable differences in the orbital evolution of the system. In addition to these equilibrium tides, we showed that imperfect initial data causes the excitation of quadrupolar modes in the neutron star, which ring with little dissipation throughout the simulation. The amplitude of these oscillations is similar to the initial amplitude of the equilibrium tide, but as it does not couple to the orbital quadrupole, or grow as the binary inspirals, its effect on the orbital evolution and waveform should be much smaller than that of the equilibrium tide.

The observable features of the merger and remnant for both types of binaries are found to be nearly impossible to distinguish. Even during merger, tidal distortion of the neutron star remains minimal, and the final masses and spins of the black holes are found to be in remarkable agreement, to $\delta M_{\mathrm{BH}}<5 \times 10^{-4} M$ and $\delta \chi_{\mathrm{BH}}<0.001$, 
respectively. The velocity kicks, although measured to only $\sim 10 \%$ accuracy, are also consistent between the two systems.

Comparisons of the gravitational wave signals show that during inspiral our simulations agree to within a phase difference $\delta \phi \sim 0.1-0.2$ rad (allowing for arbitrary time and phase shifts in the waveforms). Considering that these differences dwarf the expected tidal time shift, they represent an independent estimate of the numerical accuracy of the waveforms. At the time of merger, larger phase errors $\delta \phi \sim 1 \mathrm{rad}$ are observed. From the point of view of the Advanced LIGO detector, we find that the merger waveforms of our BBH and nondisrupting NSBH systems are extremely similar, and would be practically impossible to differentiate. Surprisingly, it thus appears that even though tidal effects during the inspiral are small for such a high mass ratio binary, they remain the largest finite size effects measurable by ground-based gravitational wave detectors. From post-Newtonian estimates and considering the Advanced LIGO detector at design sensitivity we find that, for the $q=6$ nonspinning binary considered here, a $1.4 M_{\odot}$ neutron star of radius $R_{\mathrm{NS}}=13.3 \mathrm{~km}$ would be impossible to distinguish from a black hole of the same mass for any optimally oriented event farther than $100 \mathrm{Mpc}$, neglecting degeneracies with other binary parameters. Once those degeneracies are taken into account, a more realistic requirement would be that the same optimally oriented event occurs within $\sim 30 \mathrm{Mpc}$ of the detector. These predictions are significantly more pessimistic than results obtained within the Fisher matrix formalism [20], which for some expansion parameters become unreliable when the estimated error on the size of the neutron star is $\gtrsim 15 \%$ (although a lot of useful information can still be obtained from the Fisher matrix results, if their domain of validity is verified by some direct computations of the differences between waveforms).

From these results, it is quite clear that for nonspinning binaries in the most likely range of black hole masses, NSBH and BBH binaries cannot be distinguished within the current accuracy of numerical simulations, and are extremely unlikely to be differentiable in any observable way in the immediate future. As these mergers lack the potential for post-merger electromagnetic signals (no accretion disk is formed, and no material is ejected from the system), and their gravitational wave signals will not be differentiable without significant improvements to ground-based gravitational wave telescopes, the only hope to prove the presence of a neutron star by another argument than its measured mass would be premerger electromagnetic signals (see e.g. [6-8]). Another consequence of our simulations is that, for the practical purpose of detection and parameter estimates in Advanced LIGO, NSBH in this part of the parameter space can effectively be modeled by $\mathrm{BBH}$ mergers. While numerical studies of tidal effects and neutron star disruption in lower mass ratio and/or higher spin systems remain an important task to understand the gravitational wave signals and potential electromagnetic counterparts of NSBH mergers, there appears to be little immediate incentive for further studies of low spin NSBH binaries at mass ratio $q \geqslant 5$ with general relativistic hydrodynamics codes, when more accurate solutions can be obtained at a lower computational cost by solving the binary black hole problem.

\section{ACKNOWLEDGMENTS}

The authors wish to thank Tanja Hinderer, Alessandra Buonanno and Andrea Taracchini for useful discussions regarding tidal effects in NSBH binaries, the members of the SXS collaboration for their suggestions and support over the course of this project, Ben Lackey for discussions regarding the detectability of finite-size effects in gravitational wave signals, and Andy Bohn for comments on an earlier version of this manuscript. M. D. acknowledges support through NASA Grant No. NNX11AC37G and NSF Grant No. PHY-1068243. H. P., F. F, A. M, I. M. and M. G. gratefully acknowledges support from the NSERC of Canada, from the Canada Research Chairs Program, and from the Canadian Institute for Advanced Research. L. K. gratefully acknowledges support from the Sherman Fairchild Foundation, and from NSF Grants No. PHY0969111 and No. PHY-1005426. M. S., and B. S. are partially supported by NASA ATP Grant No. NNX11AC37G and NSF Grants No. PHY-1151197, No. PHY-1068881, and No. PHY-1005655, by the Sherman Fairchild Foundation, and the Alfred P. Sloan Foundation. Computations were performed on the GPC supercomputer at the SciNet HPC Consortium [57] funded by the Canada Foundation for Innovation, the Government of Ontario, Ontario Research Fund-Research Excellence, and the University of Toronto; on Briarée from University of Montreal, under the administration of Calcul Qubec and Compute Canada, supported by Canadian Fundation for Innovation (CFI), Natural Sciences and Engineering Research Council of Canada (NSERC), NanoQuébec, RMGA and the Fonds de recherche du Québe-Nature et technologies (FRQ-NT); and on the Zwicky cluster at Caltech, supported by the Sherman Fairchild Foundation and by NSF Award PHY-0960291. 
[1] G. M. Harry (for the LIGO Scientific Collaboration), Classical Quantum Gravity 27, 084006 (2010).

[2] F. Acernese, M. Alshourbagy, P. Amico, F. Antonucci, S. Aoudia et al., Classical Quantum Gravity 25, 184001 (2008).

[3] K. Somiya (for the KAGRA Collaboration), Classical Quantum Gravity 29, 124007 (2012).

[4] M. Punturo, M. Abernathy, F. Acernese, B. Allen, N. Andersson, K. Arun, F. Barone, B. Barr, M. Barsuglia, M. Beker et al., Classical Quantum Gravity 27, 084007 (2010).

[5] M. Hannam, D. A. Brown, S. Fairhurst, C. L. Fryer, and I. W. Harry, Astrophys. J. Lett. 766, L14 (2013).

[6] D. Tsang, J.S. Read, T. Hinderer, A. L. Piro, and R. Bondarescu, Phys. Rev. Lett. 108, 011102 (2012).

[7] C. Palenzuela, L. Lehner, M. Ponce, S. L. Liebling, M. Anderson, D. Neilsen, and P. Motl, Phys. Rev. Lett. 111, 061105 (2013).

[8] V. Paschalidis, Z. B. Etienne, and S. L. Shapiro, Phys. Rev. D 88, 021504 (2013).

[9] B. D. Metzger and E. Berger, Astrophys. J. 746, 48 (2012).

[10] S. Nissanke, M. Kasliwal, and A. Georgieva, Astrophys. J. 767, 124 (2013).

[11] K. Kyutoku, K. Ioka, and M. Shibata, arXiv:1209.5747.

[12] F. Özel, D. Psaltis, R. Narayan, and J. E. McClintock, Astrophys. J. 725, 1918 (2010).

[13] L. Kreidberg, C. D. Bailyn, W. M. Farr, and V. Kalogera, Astrophys. J. 757, 36 (2012).

[14] F. Foucart, M. D. Duez, L. E. Kidder, M. A. Scheel, B. Szilágyi, and S. A. Teukolsky, Phys. Rev. D 85, 044015 (2012).

[15] F. Foucart, Phys. Rev. D 86, 124007 (2012).

[16] M. D. Duez, Classical Quantum Gravity 27, 114002 (2010).

[17] M. Shibata and K. Taniguchi, Living Rev. Relativity 14, 6 (2011).

[18] T. Damour, A. Nagar, and L. Villain, Phys. Rev. D 85, 123007 (2012).

[19] J. S. Read, L. Baiotti, J. D. E. Creighton, J. L. Friedman, B. Giacomazzo, K. Kyutoku, C. Markakis, L. Rezzolla, M. Shibata, and K. Taniguchi, Phys. Rev. D 88, 044042 (2013).

[20] B. D. Lackey, K. Kyutoku, M. Shibata, P. R. Brady, and J. L. Friedman, arXiv:1303.6298.

[21] F. Foucart, M. B. Deaton, M. D. Duez, L. E. Kidder, I. MacDonald, C. D. Ott, H. P. Pfeiffer, M. A. Scheel, B. Szilagyi, and S. A. Teukolsky, Phys. Rev. D 87, 084006 (2013).

[22] T. Hinderer, B. D. Lackey, R. N. Lang, and J. S. Read, Phys. Rev. D 81, 123016 (2010).

[23] T. Damour and A. Nagar, Phys. Rev. D 81, 084016 (2010).

[24] F. Pannarale, arXiv:1208.5869.

[25] A. Le Tiec, A. H. Mroué, L. Barack, A. Buonanno, H. P. Pfeiffer, N. Sago, and A. Taracchini, Phys. Rev. Lett. 107, 141101 (2011).

[26] Simulating eXtreme Spacetimes-a Caltech/Cornell project, http://www.black-holes.org/.

[27] L. Lindblom, M. A. Scheel, L. E. Kidder, R. Owen, and O. Rinne, Classical Quantum Gravity 23, S447 (2006).

[28] H. Friedrich, Commun. Math. Phys. 100, 525 (1985).
[29] D. Garfinkle, Phys. Rev. D 65, 044029 (2002).

[30] F. Pretorius, Classical Quantum Gravity 22, 425 (2005).

[31] M. D. Duez, F. Foucart, L. E. Kidder, H. P. Pfeiffer, M. A. Scheel, and S.A. Teukolsky, Phys. Rev. D 78, 104015 (2008).

[32] L. T. Buchman, H. P. Pfeiffer, M. A. Scheel, and B. Szilágyi, Phys. Rev. D 86, 084033 (2012).

[33] A. W. Steiner, J. M. Lattimer, and E. F. Brown, Astrophys. J. 722, 33 (2010).

[34] S. Guillot, M. Servillat, N. A. Webb, and R. E. Rutledge, Astrophys. J. 772, 7 (2013).

[35] H. P. Pfeiffer, L. E. Kidder, M. A. Scheel, and S. A. Teukolsky, Comput. Phys. Commun. 152, 253 (2003).

[36] F. Foucart, L.E. Kidder, H. P. Pfeiffer, and S. A. Teukolsky, Phys. Rev. D 77, 124051 (2008).

[37] H. P. Pfeiffer, D. A. Brown, L. E. Kidder, L. Lindblom, G. Lovelace, and M. A. Scheel, Classical Quantum Gravity 24, S59 (2007).

[38] D. A. Hemberger, M. A. Scheel, L. E. Kidder, B. Szilágyi, G. Lovelace, N. W. Taylor, and S. A. Teukolsky, Classical Quantum Gravity 30, 115001 (2013).

[39] B. Szilágyi, L. Lindblom, and M. A. Scheel, Phys. Rev. D 80, 124010 (2009).

[40] L. Lindblom, M. A. Scheel, L. E. Kidder, R. Owen, and O. Rinne, Classical Quantum Gravity 23, S447 (2006).

[41] M. Boyle and A.H. Mroué, Phys. Rev. D 80, 124045 (2009).

[42] J. Vines, É.É. Flanagan, and T. Hinderer, Phys. Rev. D 83, 084051 (2011).

[43] D. Radice, L. Rezzolla, and F. Galeazzi, arXiv:1306.6052.

[44] A. Einstein, Sitzungsber. Preuss. Akad. Wiss. Phys. Math. Kl. 47, 831 (1915).

[45] T. Damour and G. Schäfer, Nuovo Cimento Soc. Ital. Fis. 101B, 127 (1988).

[46] C. Cutler, D. Kennefick, and E. Poisson, Phys. Rev. D 50, 3816 (1994).

[47] L. Barack, T. Damour, and N. Sago, Phys. Rev. D 82, 084036 (2010).

[48] T. Hinderer, Astrophys. J. 677, 1216 (2008).

[49] B. D. Lackey, K. Kyutoku, M. Shibata, P. R. Brady, and J. L. Friedman, Phys. Rev. D 85, 044061 (2012).

[50] F. Pannarale, E. Berti, K. Kyutoku, and M. Shibata, arXiv:1307.5111.

[51] L. Barack and N. Sago, Phys. Rev. D 81, 084021 (2010).

[52] D. Shoemaker (LIGO Collaboration), Advanced LIGO Anticipated Sensitivity Curves (2010), LIGO Document T0900288-v3.

[53] L. Lindblom, B. J. Owen, and D. A. Brown, Phys. Rev. D 78, 124020 (2008).

[54] É.É. Flanagan and T. Hinderer, Phys. Rev. D 77, 021502 (2008).

[55] I. MacDonald, A. H. Mroué, H. P. Pfeiffer, M. Boyle, L. E. Kidder, M. A. Scheel, B. Szilágyi, and N. W. Taylor, Phys. Rev. D 87, 024009 (2013).

[56] B. Lackey (private communication).

[57] C. Loken, D. Gruner, L. Groer, R. Peltier, N. Bunn, M. Craig, T. Henriques, J. Dempsey, C.-H. Yu, J. Chen et al., J. Phys. Conf. Ser. 256, 012026 (2010). 